\title{
Mathematical modelling of a Fin Ray type mechanism, used in the case of the wrist rehabilitation equipment
}

\author{
Ovidiu Filip ${ }^{1, *}$, and Tudor Deaconescu ${ }^{1}$ \\ ${ }^{1}$ Department of Industrial Engineering and Management, Transilvania University of Brasov, B-dul \\ Eroilor nr. 29, Romania
}

\begin{abstract}
The paper presents the mathematical modelling of some Fin Ray-type mechanism, destined for the wrist-rehabilitation equipment, its driving being made by a pneumatic muscle. The validity of the mathematical modelling of Fin Ray mechanism is verified by threedimensional mechanism made in Pro/Engineer.
\end{abstract}

\section{Introduction}

In modern times, the successful evolution, on the international market, of a company, depends on its ability to achieve products of a high qualitative level, at low costs, which should satisfy to the highest degree, the consumers' demands [1]. This is the case of the companies manufacturing medical-rehabilitation equipment, which should keep pace with the new driving systems appeared on the market. For this purpose, a new concept is presented in this paper, of some wrist-rehabilitation equipment, based on the Fin Ray effect.

Following the accidents they suffered, many times, the patients are required to go through a period of limb immobilization. Prolonged immobilization leads to muscle wasting (hypotrophy), to dysfunctions of the circulatory system and to bone demineralization $[2,3]$. Surgical treatment of hand must be always followed by a physicalkinetic rehabilitation program [3,4]. The mobilizations shall be gently made and they aim at the patient's consciously regaining motion.

In the event the patient cannot consciously achieve the imposed movement, passive mobilization shall be resorted to. This mobilization is recommended even in the case of irreversible lesions [2].

Passive mobilization can be made in two ways:

- by the kinotherapist, through direct action on the patient;

- by means of rehabilitation equipment.

The use of the rehabilitation equipment must allow adjusting the extent of the course and the speed by which the motion of the immobile limb is achieved. Various exercises can be thereby made, at different speeds and movements, adapted to each patient's needs. These requirements are necessary in the case of the wrist-rehabilitation equipments.

* Corresponding author : ovidiu.filip@unitbv.ro 
In paper [5] several wrist-rehabilitation equipments are presented, which ensure palm motion by means of electrical or pneumatic drives. Most equipments marketed nowadays resort to electric motors for driving purposes, and ensure palm rotation in relation to the forearm. In this paper, a new pneumatically-driven rehabilitation-equipment is presented, which ensures both palm-rotation in relation to the forearm, and finger (proximal phalanges)-rotation in relation to the palm.

The palm-motion limits can be found in the literature; as follows, the values afferent to the flexion and extension of the two articulations, being presented [6]. In figure 1, the fist flexion or palmar flexion is presented, which has the maximal value of $80^{\circ}$ [6].

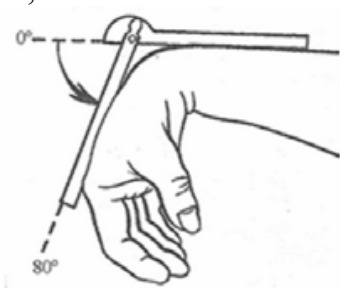

Fig. 1. Palmar flexion.

The maximal value of fist extension can be seen in figure 2 , having the value of $70^{\circ}$ [6].

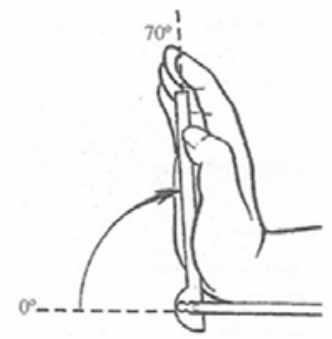

Fig. 2. Fist extension.

The following figures illustrate the finger flexion and extension. In figure 3 , it is noticeable that, in the case of flexing, the common value to all fingers is of $90^{\circ}$ [6].
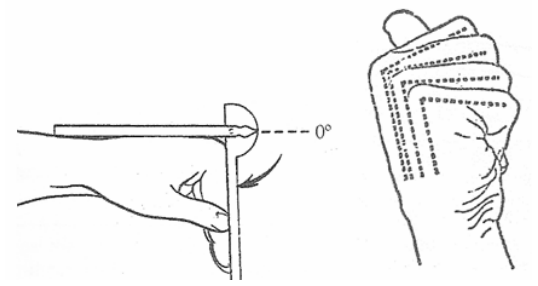

Fig. 3. Finger flexion.

Finger extension (figure 4) varies from one individual to another; and it has values ranging between $0^{\circ}$ and $45^{\circ}$ [6]. The high values of the finger extension are called hyperextension.

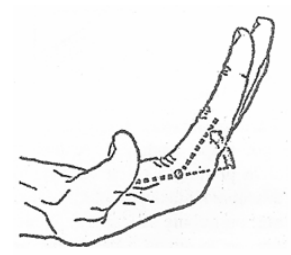

Fig. 4. Finger extension. 
Given the above presented facts, a mechanism has been designed, with a view to ensuring palm and finger motion, according to the areas indicated in the literature.

\section{Structure of the mechanism}

The wrist-rehabilitation equipment proposed in this paper, is based on a mechanism imitating the movement of fish, by means of the swim-fins. These are elastic structures crossed by thin bony fibres, which have a different behaviour from an elastic lamella, moving on the basis of the Fin Ray effect. The Fin Ray-type mechanism allowing palm movement, is shown in figure 5.

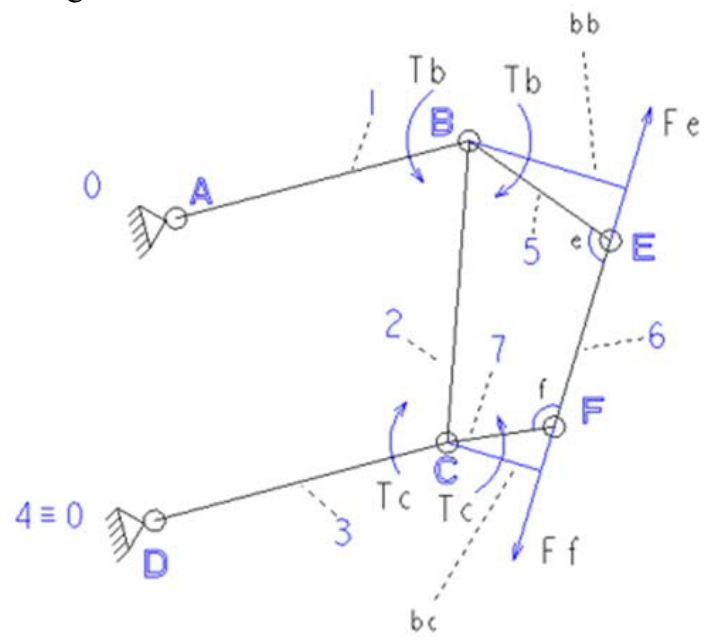

Fig. 5. Support mechanism for the palm.

The above assembly consists of two quadrilateral mechanisms. The quadrilateral mechanism $\mathrm{ABCD}$ is driven in a rotatory motion by a pneumatic muscle, by means of a cog rack-pinion mechanism. The quadrilateral mechanism BEFC is coupled to the mechanism $\mathrm{ABCD}$ by means of two torsional springs placed in the torques $\mathrm{B}$ and $\mathrm{C}$. The spring in speed torque $\mathrm{B}$ connects the arm $\mathrm{AB}$ in the first mechanism, and the arm $\mathrm{BE}$ in the second mechanism; and the spring in the torque $\mathrm{C}$ makes the connection between the arm $\mathrm{DC}$ and the arm CF.

In order to determine the mathematical model of the mechanism, the following assumptions are taken as a starting point:

- the masses of the elements will be neglected,

- the frictional forces are null,

- the elastic constant of the spring moment is the same for both torsional springs.

With a view to sizing the mechanism, the following constraints are taken as a starting point:

- the crank $\mathrm{AB}$ must rotate upwards by $70^{\circ}$ to the horizontal and by $80^{\circ}$ downwards to the horizontal;

- the lengths of the balancers $\mathrm{AB}$ and $\mathrm{BE}$ depend on the conformation of the injured palm. In this case, $\mathrm{AB}=70 \mathrm{~mm}$ and $\mathrm{BE}=40 \mathrm{~mm}$.

The other sizes of the quadrilateral mechanism were determined by simulating the operation of the mechanism, resorting to the software Pro/Engineer Wildfire 5.0. Following these simulations, the following sizes were obtained: $B C=70 \mathrm{~mm}, C D=70 \mathrm{~mm}, C F=25$ $\mathrm{mm}, \mathrm{FE}=45 \mathrm{~mm}$, the distance $\mathrm{AD}$ measured vertically, $\mathrm{ADv}=70 \mathrm{~mm}$, and horizontally $\mathrm{ADh}=5 \mathrm{~mm}$. 


\subsection{The mathematical model}

In the simulation made in Pro/Engineer Wildfire 5.0, as in reality, the position of the mechanism ABCD determines the position of the mechanism BEFC, the value of the angle $\widehat{\mathrm{DAB}}$ influencing the value of the angle $\widehat{C B E}$. In order to simplify the calculations, in the mathematical model, the angle $\widehat{\mathrm{CBE}}$ will be considered as input data, whereas the angle $\widehat{D A B}$ as output data.

Using the law of cosines in the triangles $\mathrm{ABD}$ and $\mathrm{BCD}$ (figure 5), the following relations can be written for the mechanism $A B C D$ :

$$
\begin{aligned}
& B D^{2}=A B^{2}+A D^{2}-2 A B \cdot A D \cdot \cos \widehat{D A B} \\
& A D^{2}=A B^{2}+B D^{2}-2 A B \cdot B D \cdot \cos \widehat{A B D} \\
& C D^{2}=B C^{2}+B D^{2}-2 B C \cdot B D \cdot \cos \widehat{D B C} \\
& B D^{2}=D C^{2}+B C^{2}-2 D C \cdot B C \cdot \cos \widehat{B C D} \\
& A B^{2}=A D^{2}+B D^{2}-2 A D \cdot B D \cdot \cos \widehat{A D B} \\
& B C^{2}=D C^{2}+B D^{2}-2 D C \cdot B D \cdot \cos \widehat{B D C}
\end{aligned}
$$

Identically, in the quadrilateral mechanism BEFC, using the law of cosines in the triangles $\mathrm{BCE}$ and $\mathrm{CEF}$, the following relations are obtained:

$$
\begin{aligned}
C E^{2} & =B C^{2}+B E^{2}-2 B C \cdot B E \cdot \cos \widehat{C B E} \\
C E^{2} & =E F^{2}+C F^{2}-2 E F \cdot C F \cdot \cos \widehat{C F E} \\
B C^{2} & =B E^{2}+C E^{2}-2 B E \cdot C E \cdot \cos \widehat{B E C} \\
C F^{2} & =E F^{2}+C E^{2}-2 E F \cdot C E \cdot \cos \widehat{C E F} \\
B E^{2} & =B C^{2}+C E^{2}-2 B C \cdot C E \cdot \cos \widehat{B C E} \\
E F^{2} & =C F^{2}+C E^{2}-2 C F \cdot C E \cdot \cos \widehat{E C F}
\end{aligned}
$$

So that the quadrilateral mechanism BCFE should operate properly, it is necessary that the torsional springs assembled in the torques $\mathrm{B}$ and $\mathrm{C}$ be strained over the entire working area of this mechanism $\left(90^{\circ}\right)$. Therefore, springs with arms disposed at $270^{\circ}$ (figure 6) will be used.

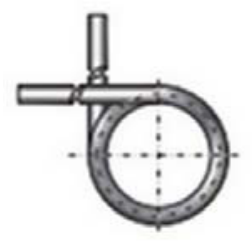

Fig. 6. Torsional spring.

The torsion moment in the torque $\mathrm{B}$ has the value:

$$
T b=F e \cdot b b=K \cdot\left(270^{\circ}-\widehat{A B E}\right)
$$

where $\mathrm{Fe}$ - force applied in the point $\mathrm{E}, \mathrm{bb}$ - arm of the force Fe, $\mathrm{K}$ - constant afferent to the spring moment.

The torsion moment in the torque $\mathrm{C}$ has the value:

$$
T c=F f \cdot b c=K \cdot\left(270^{\circ}-\widehat{D C F}\right)
$$

where $\mathrm{Fe}$ is the force applied in the point $\mathrm{E}, \mathrm{bb}-$ arm of the force Fe.

So that the crank EF should be in equilibrium, the forces Fe and $\mathrm{Ff}$ must be equal:

where:

$$
\frac{K \cdot\left(270^{\circ}-\widehat{A B E}\right)}{b b}=\frac{K \cdot\left(270^{\circ}-\widehat{D C F}\right)}{b c}
$$

$$
\begin{aligned}
& b b=B E \cdot \sin \widehat{B E F} \\
& b c=C F \cdot \sin \widehat{C F E}
\end{aligned}
$$

From the above relations, it follows:

$$
270^{\circ} \cdot(b c-b b)+b b \cdot \widehat{D C F}-b c \cdot \widehat{A B E}=0
$$


The angles $\widehat{D C F}$ and $\widehat{A B E}$ can be expressed as a sum of angles which may be determined from the two quadrilateral mechanisms:

$$
\begin{aligned}
& \widehat{A B E}=\widehat{A B C}+\widehat{C B E}=\widehat{A B D}+\widehat{D B C}+\widehat{C B E} \\
& \widehat{D C F}=\widehat{B C D}+\widehat{B C F}=\widehat{D C B}+\widehat{B C E}+\widehat{E C F}
\end{aligned}
$$

Given that the movement of entry into the mechanism for the support of the palm, in the case of this analysis, is the rotation of the arm BE it follows that the angle $\widehat{\mathrm{CBE}}$ is known. By attributing different values to the angle $\widehat{C B E}$ the positions of the mechanism BCEF can be determined.

The connection between the mechanism BCEF and the mechanism ABCD is achieved by the equilibrium of the forces, at the level of the crank EF. In this respect, the angles $\widehat{A B D}, \widehat{D B C}$ and $\widehat{B C D}$ become:

$$
\begin{aligned}
& \widehat{\mathrm{ABD}}=\cos ^{-1}\left(\frac{\mathrm{AB}^{2}-\mathrm{AD}^{2}+\mathrm{AB}^{2}+A D^{2}-2 \mathrm{AB} \cdot \mathrm{AD} \cdot \cos \widehat{\mathrm{DAB}}}{2 \mathrm{AB} \cdot \sqrt{\mathrm{AB}^{2}+\mathrm{AD}^{2}-2 \mathrm{AB} \cdot \mathrm{AD} \cdot \cos \widehat{\mathrm{DAB}}}}\right) \\
& \widehat{\mathrm{DBC}}=\cos ^{-1}\left(\frac{\mathrm{BC}^{2}-\mathrm{CD}^{2}+\mathrm{AB}^{2}+\mathrm{AD}^{2}-2 \mathrm{AB} \cdot \mathrm{AD} \cdot \cos \widehat{\mathrm{DAB}}}{2 \mathrm{BC} \cdot \sqrt{\mathrm{AB}^{2}+\mathrm{AD}^{2}-2 \mathrm{AB} \cdot \mathrm{AD} \cdot \cos \overline{\mathrm{DAB}}}}\right) \\
& \widehat{\mathrm{BCD}}=\cos ^{-1}\left(\frac{\mathrm{DC}^{2}+\mathrm{BC}^{2}-\mathrm{AB}^{2}-\mathrm{AD} \mathrm{D}^{2}+2 \mathrm{AB} \cdot \mathrm{AD} \cdot \cos \overline{\mathrm{DAB}}}{2 \mathrm{DC} \cdot \mathrm{BC}}\right)
\end{aligned}
$$

By introducing the relations 19 and 20 in the relation 18 , there is obtained:

$$
270^{\circ} \cdot(b c-b b)+b b \cdot(\widehat{B C D}+\widehat{B C F})-b c \cdot(\widehat{A B D}+\widehat{D B C}+\widehat{C B E})=0
$$

By introducing in the relation 24, the relations 21, 22, 23, an equation is obtained, having $\cos \widehat{D A B}$ as unknown. The equation is solved with Matlab software.

\subsection{Matlab application used to determine the angles of the mechanism}

As follows, a Matlab application is presented, by which $\widehat{D A B}$ is calculated, resorting to different values of the angle $\widehat{\mathrm{CBE}}$ as input data.

The calculation software for the angle $\widehat{D A B}$

$\%$ arms mec 1

$11=70$

$12=70$

$13=70$;

$14=\operatorname{sqrt}\left(70^{\wedge} 2+5^{\wedge} 2\right)$

$\%$ arms mec 2

$12=70$;

$15=40$;

$16=45$;

$17=25$;

disp ('type the angle $u b=$ ')

disp ('then the secondary quadrilateral mechanism will be calculated')

disp ('type "calc"')

$\mathrm{b}=$ degtorad $(\mathrm{ub})$

$\mathrm{d} 2=\operatorname{sqrt}\left((12)^{\wedge} 2+(15)^{\wedge} 2-2 * 12 * 15 * \cos (b)\right)$;

$\mathrm{e} 1=\operatorname{acos}\left(\left((15)^{\wedge} 2+(\mathrm{d} 2)^{\wedge} 2-(12)^{\wedge} 2\right) /\left(2^{*} 15^{*} \mathrm{~d} 2\right)\right)$;

$\mathrm{e} 1 \mathrm{~g}=\operatorname{radtodeg}(\mathrm{e} 1)$;

$\mathrm{e} 2=\operatorname{acos}\left(\left((16)^{\wedge} 2+(\mathrm{d} 2)^{\wedge} 2-(17)^{\wedge} 2\right) /\left(2 * 16^{*} \mathrm{~d} 2\right)\right)$;

$\mathrm{e} 2 \mathrm{~g}=\operatorname{radtodeg}(\mathrm{e} 2)$;

$\mathrm{c} 1=\operatorname{acos}\left(\left((12)^{\wedge} 2+(\mathrm{d} 2)^{\wedge} 2-(15)^{\wedge} 2\right) /(2 * 12 * \mathrm{~d} 2)\right)$;

$\mathrm{c} 1 \mathrm{~g}=\operatorname{radtodeg}(\mathrm{c} 1)$;

$\mathrm{c} 2=\operatorname{acos}\left(\left((17)^{\wedge} 2+(\mathrm{d} 2)^{\wedge} 2-(16)^{\wedge} 2\right) /(2 * 17 * \mathrm{~d} 2)\right)$;

$\mathrm{c} 2 \mathrm{~g}=\operatorname{radtodeg}(\mathrm{c} 2)$; 


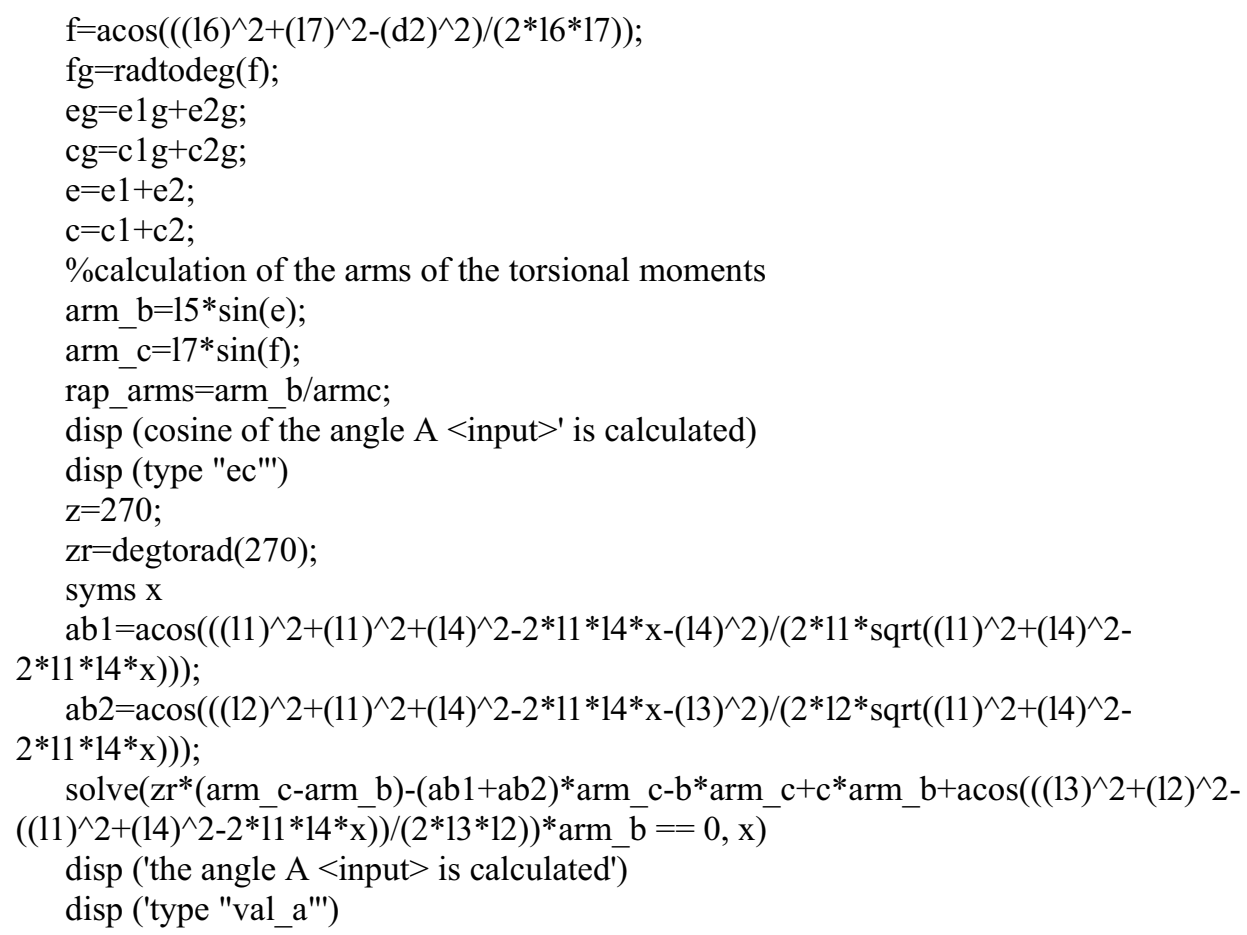

The mathematical model was verified by using the obtained data in the simulation of the mechanism achieved in Pro/Engineer.

Following the simulation of the mechanism, several positions of this one were obtained. 16 positions were retained, which describe the entire course of $150^{\circ}$ to be covered by the mechanism, so as to ensure the movement of the palm between the maximal values of the fist flexion and extension. In these positions, the values of the angles $\widehat{\mathrm{DAB}}$ and $\widehat{\mathrm{CBE}}$ were measured.

Introducing the values of the angle $\widehat{C B E}$ (variable $u b$ in the application) the values of the angle $\widehat{D A B}$ (variable val_a in the application) will be obtained.

The obtained values in Pro/Engineer and in Matlab are presented in figure 7.
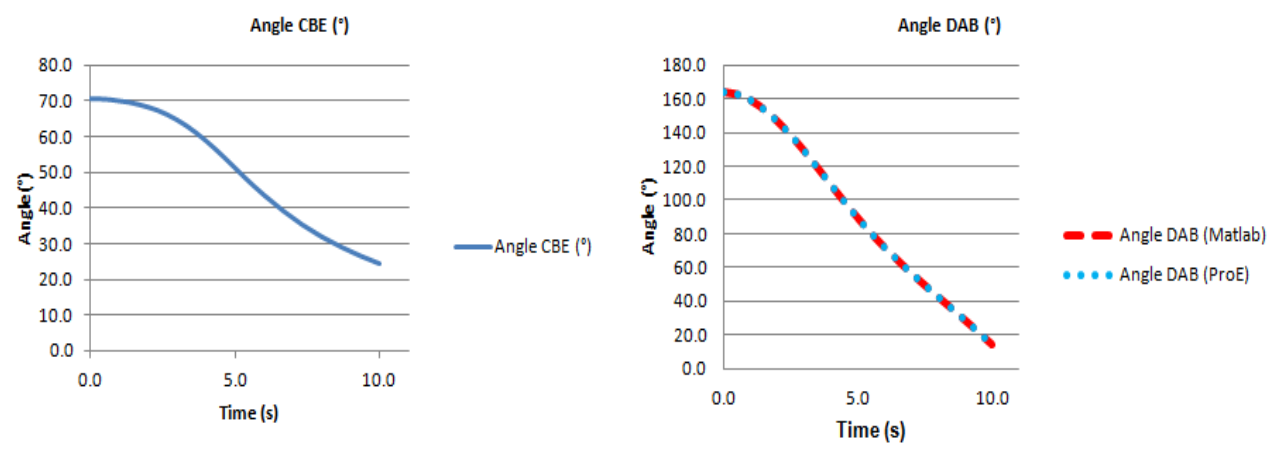

Fig. 7. Values of the angles $\widehat{\mathrm{CBE}}$ and $\widehat{\mathrm{DAB}}$.

Small differences (at the level of $0.000 \mathrm{x}$ degrees) can be noticed between the two values of the angle $\widehat{\mathrm{DAB}}$ obtained in Pro/Engineer and in Matlab.

The values obtained in Pro/Engineer are presented in figure 8. 


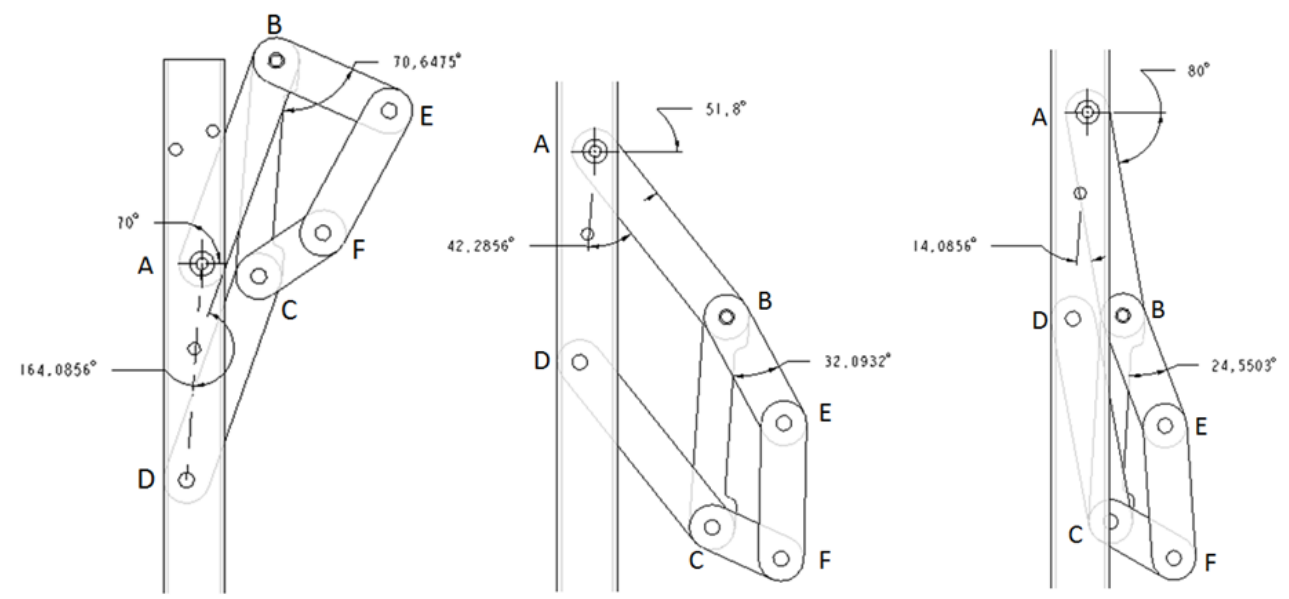

Fig. 8. Values of the angles $\widehat{\mathrm{DAB}}$ and $\widehat{\mathrm{CBE}}$ obtained in Pro/Engineer.

Figure 8 also shows that this mechanism can be used for mobilizing the fingers over their active area, between the maximal-flexion $\left(90^{\circ}\right)$ and the maximal-extension areas, ranging between $\left(0^{\circ}-45^{\circ}\right)$.

\section{Conclusions}

As a result of the facts presented above, the following ideas are worth keeping in mind:

- the presented mechanism ensures palm motion over its entire active area;

- this mechanism can be used also for the finger (proximal phalanges) motion, over their active area;

- a mathematical model was designed, which is validated by the simulation in Pro/Engineer;

- a Matlab application was designed, in order to determine the angle $\widehat{\mathrm{DAB}}$.

- in the future, this mechanism can be used for the wrist-rehabilitation equipment.

\section{References}

1. A. Deaconescu, RMEE, 14, 1, 29-38, (2015)

2. C. Albu, T.L. Vlad, A. Albu, Passive kinesiotherapy (Editura Polirom, Iași, 2004)

3. O. Filip, T. Deaconescu, Advances in Production, Automation and Transportation System, Proceedings of the $6^{\text {th }}$ International Conference on Manufacturing Engineering, Quality and Production Systems (MEQAPS '13), Proceedings of the $4^{\text {th }}$ International Conference on Automotive and Transportation Systems (ICAT '13), 5559, (2013)

4. I. Kiss, Physio-kinesiotherapy and medical recovery (Editura Medicala, București, 2012)

5. O. Filip, T. Deaconescu, ICEEMS, Recent, 14, 4, 263-266, (2013)

6. E.L. Sidenco, Practical guide for joint and muscle evaluation, (Editura Fundației „Romania de Maine”, București, 2009) 